\section{S13 ANTIBIOTIC PENETRATION INTO THE INFECTED PLEURAL SPACE; A PK/PD STUDY}

${ }^{1}$ DT Arnold, ${ }^{2} \mathrm{~L}$ Read, ${ }^{2} \mathrm{~A}$ Noel, ${ }^{1} \mathrm{FW}$ Hamilton, ${ }^{2} \mathrm{~A}$ MacGowan, ${ }^{2} \mathrm{M}$ Bayliss, ${ }^{1} \mathrm{~N}$ A Maskell. ${ }^{1}$ Academic Respiratory Unit, Bristol, UK; ${ }^{2}$ Antimicrobial Reference Laboratory, Bristol, UK

10.1136/thorax-2021-BTSabstracts. 19

Introduction and Objectives Pleural infection is a serious clinical condition with an average hospital length of stay of 13 days. Current standard of care defaults to hospital admission for drainage of the infected fluid and intravenous antibiotics. The standard empirical antibiotic choice varies nationally but the ability of these antibiotics to reach the pleura is poorly understood. Previous pharmacokinetics and pharmacodynamics (PK/PD) literature on the penetrance of antibiotics into pleural fluid is extrapolated from non-infected effusions (e.g. malignant effusions) or animal models.

The Pleural Antibiotic Concentrations informing Treatment (PACT) study is a single centre PK/PD study that aims to assess the concentration of antibiotics within the infected pleural space to improve the evidence base around antimicrobial choice, route and duration of therapy.

Methods Patients with parapneumonic effusions/empyema planned for pleural drainage were prospectively recruited. Serial pleural fluid samples were collected timed with routine antibiotic administration and paired with synchronous serum sampling. Pleural fluid and serum antibiotic concentrations were measured using a validated high performance liquid chromatography (HPLC) method at the National Antimicrobial Reference Laboratory (Bristol UK).

Results This study is ongoing, further results on a wider range of antimicrobials will be available for the conference.

At the time of writing, 18 patients had been recruited (15 CPPE, 3 empyema) with over 150 paired serum/fluid samples collected. Ten different antibiotics have been assayed although the majority of timepoints relate to Amoxicillin/Co-amoxiclav $(\mathrm{n}=36)$, Metronidazole $(\mathrm{n}=28)$ and Piperacillin-Tazobactam $(n=22)$ at this time, see figure 1 .

For these antibiotics the peak concentration and area under the curve within the pleural space was equivalent to serum levels. Importantly, across the dosing schedule the pleural antibiotic levels never fell below the minimum inhibitory concentrations (MICs) for bacteria known to cause pleural infection even when given orally. The pleural levels for penicillins persisted beyond the dosing schedule ( 8 hours) and were not affected by pleural $\mathrm{pH}$ or fibrinolytics.

Conclusions For 3 commonly used antibiotics (Amoxicillin, Metronidazole, Piperacillin-Tazobactam) the pleural fluid concentration of antibiotic remined well above the usual MICs of causative bacteria. Penetrance and persistence of antibiotics make twice-daily or oral administration a possibility in pleural infection.

\section{S14 LENGTH OF ANTIBIOTIC COURSE FOR TREATING PLEURAL INFECTION: A RANDOMISED TRIAL}

M Hassan, M Gadallah, B El-Shaarawy, A El-Shazly, AS Sadaka. Chest Diseases Department, Alexandria University Faculty of Medicine, Alexandria, Egypt

\subsection{6/thorax-2021-BTSabstracts.20}

Background Expert opinion recommends treating pleural infection with at least 4 weeks (and up to 6 weeks) of antibiotics and stronger evidence is required to guide management. We aim in this trial to investigate whether shorter durations of antibiotic therapy are as effective as (standard) longer courses. Methods The short vs long antibiotic course for pleural infection management (SLIM) randomised open-label trial aims to recruit 50 patients with pleural infection randomised 1:1 to receive a short course (total duration of 2-3 weeks, group A) or long course (4-6 weeks, group B) of antibiotics. Patients are included if they are adults hospitalised for pleural infection, at low-moderate risk of mortality (RAPID score 0-4), who have been stabilized and are ready to be shifted to oral therapy within 14 days of admission. The primary endpoint is infection relapse six weeks post admission (defined as worsened pleural collection on imaging with elevation of post-discharge inflammatory markers) in the study groups. Secondary outcomes include length of antibiotic courses and time to return to normal daily activity in study groups. Recruitment to trial is ongoing.

Results Between Oct 2020 and June 2021 thirty-six patients were recruited; 18 patients randomised to each group. Follow up data was available for 17 patients in each group (one loss to follow up and one death before follow-up visit). Table 1 summarises data of study patients. By 6 weeks post admission $3 / 17(17.6 \%)$ of group A patients and 2/17 (11.7\%) of group $B$ patients had relapse of infection $\left(\chi^{2} 0.234, p=0.628\right)$. All five relapses occurred while patients were still on antibiotics. After controlling for RAPID score, the adjusted odds ratio of relapse with shorter antibiotic courses was 1.301 (95\% CI 0.171 to $9.891, \mathrm{p}=0.800$ ).

The mean difference in the length of antibiotic treatment between the 2 groups was 13.2 days (95\% CI 10.7 to 15.7 days). 8/13 (62\%) of group A and 8/16 (50\%) of group B patients reached normal level of activity by six weeks post diagnosis.

Conclusion In adult patients with pleural infection and lowmoderate RAPID score who are stabilized within 14 days of
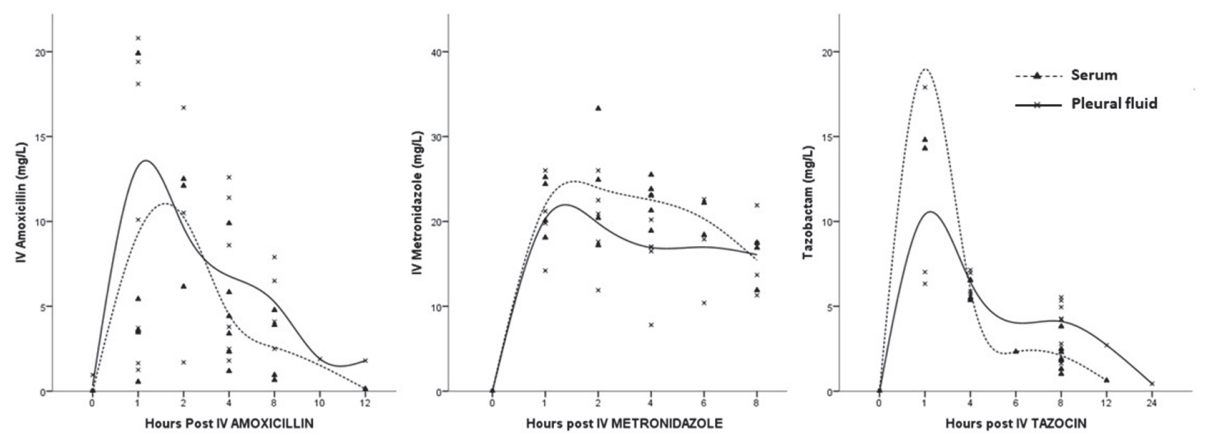

Abstract S13 Figure 1 Amoxicillin, Metronidazole and Tazocin (Tazobactam) levels in serum and pleural fluid post intravenous administration 